\title{
Pesquisa em treinamento de força no Brasil: análise dos grupos e produção científica
}

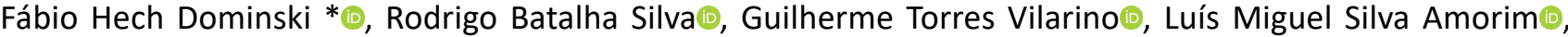 \\ Alexandro Andrade
}

\section{Palavras-chave: \\ Treinamento de resistência; \\ Conhecimento; \\ Pesquisa; \\ Força muscular.}

\section{Keywords:}

Resistance training;

Knowledge;

Research;

Muscle strength.

\begin{abstract}
RESUMO
Este estudo objetivou analisar a distribuição geográfica, evolução e produção científica dos grupos de pesquisa (GP) em treinamento de força no Brasil. Trata-se de um estudo descritivo, de análise documental, feito na base de dados do Diretório dos GP do Brasil do CNPq. Foi feita análise das produções cientificas dos líderes dos GP entre 2014 a 2018. Foram localizados 41 GP. A maioria dos GP está localizada no Estado de São Paulo. As publicações se concentram no estrato A1 e com uma expressiva tendência nos estratos de maior qualidade ao longo dos anos. A maioria dos estudos investigou praticantes de musculação, seguido por idosos. Observa-se que os GP brasileiros sobre treinamento de força apresentam produção científica de qualidade.
\end{abstract}

\section{Palavras Chave:}

Entrenamiento de resistencia; Conocimiento; Investigación; Fuerza muscular.

\begin{abstract}
This study aimed to analyze the geographic distribution and scientific production of research groups (RG) of strength training in Brazil. This is a descriptive and systematic study realized on the current database Directory of RG in Brazil available on the website of the CNPq. We analyze the scientific productions of the leaders of the RG between 2014 to 2018. There were 41 RG in the area. Most of the RG are located in São Paulo. The publications are concentrated in stratum A1 and have shown with an expressive trend of higher quality over the years. Most of the studies investigated strength training practitioners, followed by elderly. Brazilian RG that investigate strength training produce relevant knowledge and the quality of scientific production is evolving.
\end{abstract}

\section{RESUMEN}

En este estudio se pretende analizar la distribución geográfica, la evolución y la producción científica de los grupos de investigación (GI) sobre fortalecimiento muscular en Brasil. Para ello se realiza un estudio sistemático y descriptivo, de análisis documental, sobre la base de datos del Directorio de los GI de Brasil del CNPq. Se analizó la producción científica de los directores de los GI entre 2014 y 2018. Se localizó a 41 GI. La mayoría de los GI se encuentra en el estado de São Paulo. Las publicaciones se concentran en el estrato A1 y con una expresiva tendencia en los estratos de mayor calidad a lo largo de los años. En la mayoría de los estudios se investigó a los practicantes de musculación, seguido de las personas mayores. Se constata que los GI brasileños sobre fortalecimiento muscular cuentan con una producción científica de calidad.

Universidade do Estado de Santa Catarina (Udesc), Centro de Ciências da Saúde e do Esporte (Cefid), Programa de Pós-Graduação em Ciências do Movimento Humano, Florianópolis, SC, Brasil.

*Autor correspondente:

Fábio Hech Dominski

E-mail: fabiohdominski@hotmail.com

Recebido em 1 de dezembro de 2018; aceito em 14 de fevereiro de 2019.

DOI: 10.1016/j.rbce.2019.02.002 


\section{INTRODUÇÃO}

O treinamento de força (TF) refere-se a uma intervenção em que os praticantes submetem um músculo ou grupos musculares a uma resistência externa (Esco, 2013), com o objetivo de aumentar a força, potência, hipertrofia e desempenho motor (Westcott, 2009; Mynarski et al., 2014), através de uma variedade de manifestações, como treinamento com pesos livres, aparelhos de musculação, peso corporal, entre outros (Rhodes et al., 2017).

A prática regular do TF é recomendada para a população em geral, é sugerido pelo American College of Sports Medicine que o treinamento seja feito por pelo menos duas vezes por semana com duração de 30 minutos cada seção (ACSM, 2009). Entre os benefícios advindos da prática do TF, podemos destacar a redução na taxa de mortalidade, controle do peso, pressão arterial e diabetes e aumento da qualidade de vida (Drenowatz et al., 2015; Kraschnewski et al., 2016; Casonatto et al., 2016; Ishiguro et al., 2016). Diante dos inúmeros benefícios verificados em estudos e da facilidade de acesso da população, o TF está há anos entre as principais tendências fitness segundo o Colégio Americano de Medicina do Esporte (Thompson, 2017).

A produção de conhecimento sobre o TF está em constante evolução. Os primeiros estudos foram publicados por volta de 1890 (Kraemer et al., 2017). Observa-se que os primeiros especialistas que atuavam na área prática usavam técnicas oriundas de suas experiências empíricas, não tinham respaldo teórico e/ ou científico (Kraemer et al., 2017). Atualmente, notase um aumento da produção de conhecimento sobre $o$ TF, principalmente proveniente dos grupos de pesquisa (GP). Os GP são caracterizados como um conjunto de indivíduos organizados hierarquicamente em torno de líderes acadêmicas, para a execução de atividades coletivas ou compartilhadas.

$\mathrm{Na}$ área da educação física as pesquisas científicas no Brasil tiveram início na década de 1970 (Lazzarotti Filho et al., 2018). No contexto brasileiro, os grupos de pesquisa são registrados no Diretório dos Grupos de Pesquisa no Brasil (DGPB) do Conselho Nacional de Desenvolvimento Científico e Tecnológico (CNPq). Nessa base de dados constam informações sobre as linhas de pesquisa dos grupos, os recursos humanos e as parcerias entre as instituições às quais os grupos pertencem. De acordo com o último Censo do DGPB feito em 2016, existem mais de 37 mil grupos de pesquisa no país.

Estudos que analisam as características dos GP que investigam o TF permitem maior entendimento e conhecimento em relação ao desenvolvimento científico da área. A análise da produção científica dos
GP, assim como sua distribuição geográfica, possibilita a identificação de lacunas e potencias assuntos a serem explorados em pesquisas futuras. De forma mais específica, esse tipo de estudo permite a identificação dos pesquisadores e as universidades efetivamente engajadas na pesquisa (Ferraz et al., 2018). Nesse sentido foram desenvolvidos estudos com tais análises em relação às áreas de psicologia do esporte (Vilarino et al., 2017), Cineantropometria (Santos et al., 2011) e modalidades esportivas (Marinho e BarbosaRinaldi, 2010; Teixeira e Marinho, 2010). Entretanto, não foram observadas análises sobre os GP em TF no país.

Devido à pluralidade de formas em que o TF se manifesta e como o Brasil é o $2^{\circ}$ país em número de academias de musculação no mundo, com mais de 34 mil academias e cerca de nove milhões de praticantes, a hipótese é que exista um elevado número de GPs que investiguem o tema, com produção de conhecimento sobre diversas populações.

Nesse contexto, o objetivo do estudo foi analisar a distribuição geográfica, evolução e produção científica dos grupos de pesquisa em treinamento de força no Brasil.

\section{MATERIAL E MÉTODOS}

O presente estudo caracteriza-se como sistemático e descritivo, de análise documental, feito na base de dados do Diretório dos Grupos de Pesquisa do Brasil (DGPB) do Conselho Nacional de Desenvolvimento Científico e Tecnológico (CNPq) sobre a temática treinamento de força (Conselho, In pressa).

Os termos usados para busca na base corrente do DGPB foram "Treinamento de força", "Treinamento resistido", "Treinamento de resistência", "Exercício resistido" e "Musculação" e foram selecionados os seguintes campos: "nome do grupo" e "nome da linha de pesquisa". Como critério de inclusão adotouse a certificação dos grupos de pesquisa, excluíramse os grupos não atualizados. Tal autenticação é de responsabilidade dos dirigentes das atividades de pesquisa da instituição à qual o líder do grupo está vinculado. Não foram aplicados filtros na busca em relação à localização, ao tempo de existência do grupo, à área do conhecimento, ao setor da aplicação, à formação acadêmica, aos bolsistas CNPq ou docentes. A busca foi encerrada em setembro de 2018.

Foram considerados como grupos específicos (GE) de pesquisa em TF aqueles que apresentassem algum dos termos usados para busca no nome do grupo. Os demais grupos foram classificados como não específicos (GNE).

As análises foram feitas a partir da extração das seguintes informações dos grupos de pesquisa: ano de 
formação, área de conhecimento, tipo de instituição de ensino superior (pública ou privada), unidade da federação, região demográfica, linhas de pesquisa e número de integrantes (professores, estudantes, técnicos e colaboradores estrangeiros). Para a análise da produção científica dos GP foi verificado o currículo do líder de pesquisa de cada grupo através da Plataforma Lattes do CNPq (Conselho, In pressb). Para caracterização da produção científica dos líderes dos grupos foi delimitada a produção de artigos publicados mais recentemente, compreendida no período dos últimos cinco anos (janeiro de 2014 até setembro de 2018). Tal delimitação justificase por ser uma produção considerada atual e por ser o período de tempo usado por agências de fomento (CNPq) para avaliação da produção dos pesquisadores e grupos. Foram considerados para a análise apenas artigos completos e artigos aceitos sobre treinamento de força. A classificação dos artigos foi feita de acordo com o Qualis periódicos da Plataforma Sucupira - classificação de periódicos quadriênio 2013-2016 (Coordenação, 2013a), na área de avaliação educação física. Esse documento classifica os periódicos nos estratos $A 1, A 2, B 1$, B2, B3, B4, B5 e C. Também foram considerados os artigos publicados em periódicos não classificados. A partir da produção científica dos GP dos artigos completos publicados em periódicos, analisaram-se o número total de artigos, a qualidade da produção, a população investigada e o tema, através da extração das palavras-chave citadas pelos autores, além do líder de GP mais produtivo. Foram consideradas as palavras-chave no idioma original de publicação de cada artigo e foi feito o agrupamento dos sinônimos, como os termos "strength training" e "treinamento de força".

\section{RESULTADOS}

Após feita a busca no DGPB com os termos e de acordo com os critérios de inclusão adotados, foram localizados 41 GP cadastrados. Dos 41 GP, 17 foram classificados como GP específicos (GE) e 24 não específicos (GNE) (Tabela 1).

\section{Ano de formação dos GP}

O registro do primeiro grupo de pesquisa ocorreu em 1995 - Grupo Ciências Fisiológicas, na Universidade Federal Rural do Rio de Janeiro. A respeito da formação dos grupos de pesquisa, destaca-se 2010, com a formação de sete grupos de pesquisa em TF. A Figura 1 mostra o crescimento na formação dos grupos de pesquisa, de 1995 até 2018.

\section{Distribuição geográfica dos GP}

Foram localizados grupos de pesquisa em 15 estados. A maioria dos grupos de pesquisa $(n=$ 10/24,3\%) está localizada no Estado de São Paulo, seguido de Minas Gerais e Rio de Janeiro ( $n=5$ cada/12,1\%) (Figura 2). A Região Sudeste concentra o maior número de grupos em TF, com $22(53,6 \%)$. Há ausência de GP em TF nos estados do Acre, Alagoas, Amazonas, Amapá, Bahia, Goiás, Mato Grosso, Mato Grosso do Sul, Maranhão, Roraima, Rondônia, Rio Grande do Norte e Tocantins. Assim, as regiões Norte e Centro-Oeste são as mais carentes em relação à presença de GP, conforme ilustra a Figura 2.

\section{Características dos GP}

A maior frequência observada de GP provém de instituições públicas ( $n=30 / 73,1 \%$ ), em relação às instituições privadas ( $n=11 / 26,9 \%$ ). Quanto aos recursos humanos, observaram-se 687 integrantes atuantes na área, 267 pesquisadores, 404 estudantes, sete técnicos e nove colaboradores estrangeiros. Além disso, foi observado um número de 226 egressos dos $\mathrm{GP}$, entre pesquisadores e estudantes. Desse total de pesquisadores dos GP, $312(45,4 \%)$ têm ou cursam doutorado.

Foram encontradas 29 instituições com GP que investigam o TF. A Universidade Federal do Maranhão (UFMA) apresentou três grupos, outras cinco instituições têm registrados dois grupos e o restante das instituições um grupo. Vinte instituições têm parcerias interinstitucionais, que variam de uma até oito instituições parceiras.

Observa-se que a área predominante de conhecimento dos GP foi ciências da saúde, relacionada à educação física. Os GP apresentaram 157 linhas de pesquisa, variavam de uma até 18 linhas de pesquisa por grupo. A maioria dos grupos tem possui de uma a três linhas de pesquisa (Tabela 2).

\section{Produção científica dos GP}

De 2014 a 2018 foram produzidos 431 artigos pelos líderes dos GP brasileiros vinculados a área de TF, 264 (61,25\%) estudos publicados por GE e 167 $(38,75 \%)$ publicados por GNE. Foi observado que os GP que investigam o tema publicaram principalmente no estrato A1 ( $n=166 / 38,5 \%$ ) nos últimos cinco anos (Figura 3). O líder do Grupo de Pesquisa em Treinamento de Forçada Universidade Federal do Rio de Janeiro (UFRJ) apresentou o maior número de artigos publicados sobre o tema no período analisado $(n=63), 16$ estudos classificados no extrato A1. O 
Tabela 1. Grupos de pesquisa sobre treinamento de força cadastrados no DGPB.

Efeitos histomorfométricos, ultraestruturais e imuno-histoquimicos nos órgãos e tecidos de ratas ovariectomizadas com diferentes fontes proteicas na dieta alimentar submetidas a exercício resistido

Fisiologia do exercício aeróbio e resistido para a saúde e o alto rendimento

Fundamentos do treinamento de força e prescrição do exercício físico

Grupo de Estudo e Pesquisa em Adaptações Neuromusculares ao Treinamento de Força

UniCEUB

Grupo de Estudo e Pesquisa em Treinamento de Força (GEStrength)

IFCE

USP

Grupo de Estudo em Treinamento de Força

Grupo de Estudo em Treinamento de Força e Fisiologia do Exercício

UEL

Grupo de Estudos e Pesquisa do Treinamento na Musculação

Uniararas

UFES

Grupos

específicos

Grupo de Estudos e Pesquisas em Avaliação e Musculação (GEPAM)

UFMG

UFOP

Grupo de Estudos e Pesquisas em Treinamento de Força (LabFor)

UFJF

Grupo de Pesquisa em Cineantropometria, Performance Humana e Treinamento de Força

UFRRJ

Grupo de Pesquisa em Metabolismo, Nutrição e Treinamento de Força (GPMENUTF)

UFPR

Grupo de Pesquisa em Musculação e Treinamento de Força

Grupo de Pesquisa em Treinamento de Força

Claretiano

UFRJ

Grupo de Pesquisa em Treinamento de Força (GPTF)

UFRGS

Investigação de alterações morfológicas e funcionais nos órgãos do trato urinário inferior induzidas pelo

exercício resistido dinâmico em ratas nulíparas, multíparas e ovariectomizadas

USJT

Manipulação das variáveis metodológicas do treinamento de força, periodização e suas influências

no volume de treinamento, desempenho, ativação muscular e alterações hormonais

Atividade Física, Esporte e Saúde

UFRJ

Biodesa - Grupo de Pesquisa em Biodinâmica do Desempenho, Exercício e Saúde

Uepa

Ciências fisiológicas

UCB

UFRRJ

Fisiologia e epidemiologia do exercício e da atividade física

UnB

Grupo de Estudo e Pesquisa em Exercício Físico e Adaptações Neuromusculares (Gepefan)

Unicamp

Grupo de Estudo e Pesquisa em Fisiologia e Prescrição do Exercício

UFMA

Grupo de Estudo e Pesquisa em Imunometabolismo do Músculo Esquelético e Exercício

UFPI

Grupo de Estudos e Pesquisa em Exercício Físico e Desempenho Esportivo

UFPE

Grupo de Pesquisa em Adaptações Biológicas ao Exercício Físico

USP

Grupo de Pesquisa em Atividade Física e Saúde

específicos

Grupo de Pesquisa em Atividade Física e Saúde (GPAFS)

IFF

Unesc

Grupo de Pesquisa em Biomecânica e Fisiologia do Exercício

Unipac

UFPB

Grupo de Pesquisa em Treinamento e Avaliação do Desempenho Humano

Unig

Grupo Select de Pesquisa e Treinamento Profissional

UFMA

Laboratório de Biologia Celular e Molecular do Músculo Esquelético (Labcemme)

Univasf

Laboratório de Nutrição e Treinamento Esportivo (LAN)

UFMG

Núcleo de Pesquisa em Adaptações Neuroimunoendócrinas ao Exercício (Nano)

UFMA

IES - Instituição de Ensino Superior.

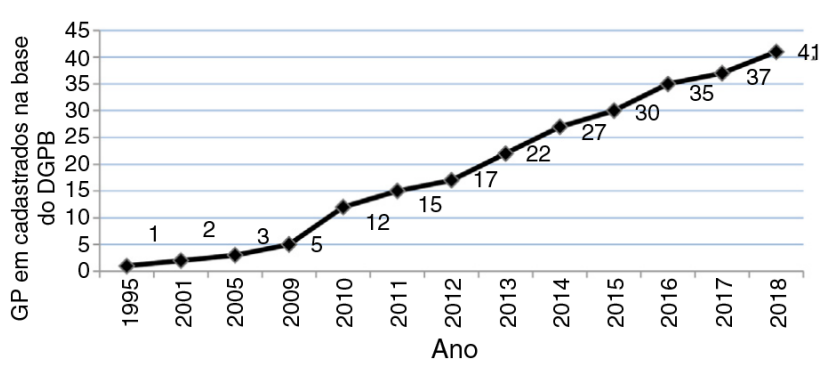

Figura 1. Ano de formação dos grupos de pesquisa no Brasil relacionados a treinamento de força. Legenda: Número total de GP em TF acumulados. líder do Grupo de Estudo e Pesquisa em Adaptações Neuromusculares ao Treinamento de Força da Universidade de São Paulo (USP) foi o pesquisador com maior quantidade de artigos no estrato A1, com 37 , o que corresponde a $86 \%$ da sua produção no último quinquênio.

$\mathrm{Na}$ análise por grupos específicos e não específicos, observa-se que, apesar de em menor número de GP, os GE apresentam maior número de artigos em todos os estratos quando comparados com os GNE (Figura 4). 


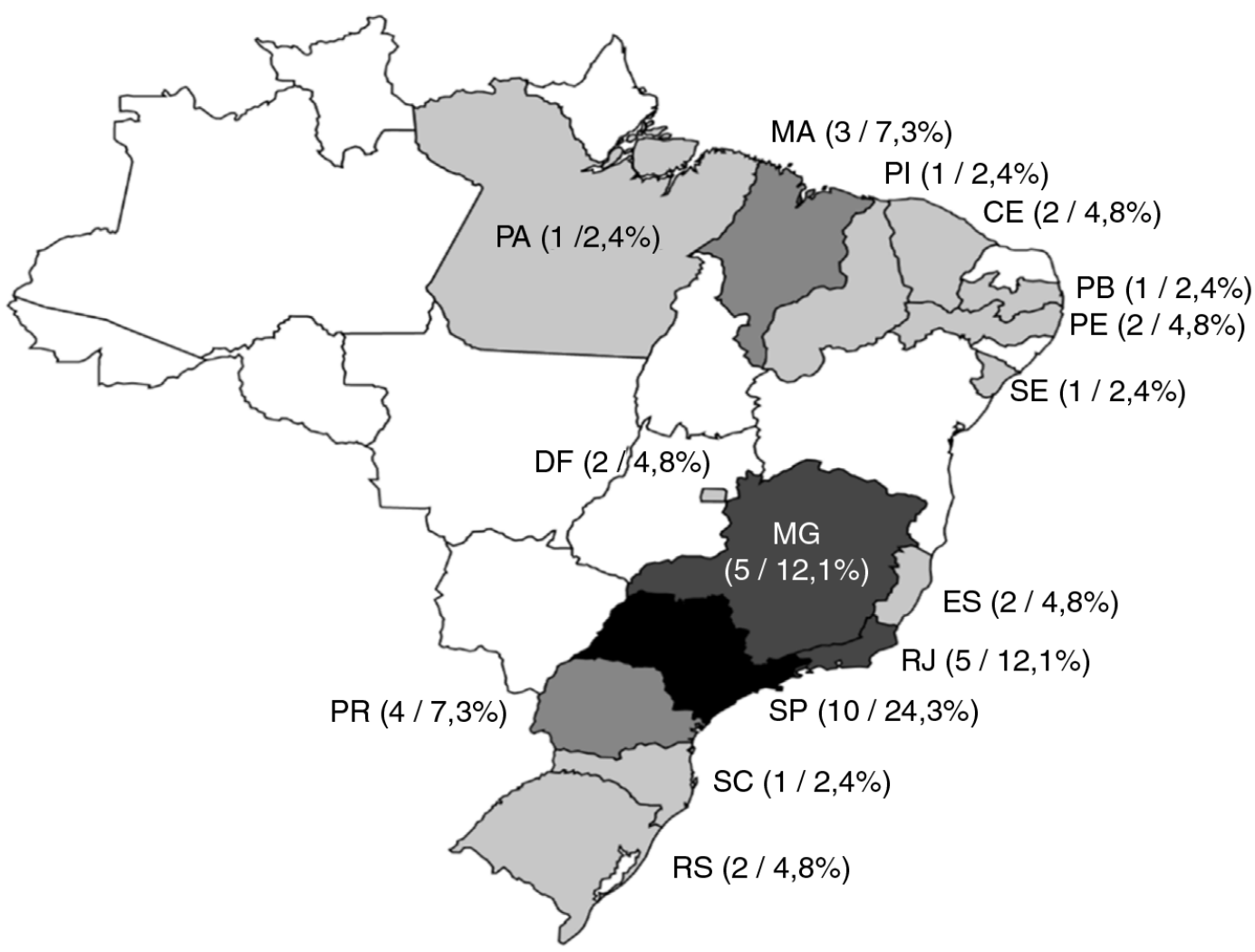

Figura 2. Distribuição geográfica dos grupos de pesquisa em TF no Brasil. Legenda: Quanto mais escura a cor do estado, maior número de GP. Os estados em branco não apresentaram GP em TF no DGPB.

Tabela 2. Distribuição dos integrantes, instituições, área de conhecimento e linhas de pesquisa dos grupos de pesquisa em TF.

\begin{tabular}{lcc}
\hline Características dos grupos de pesquisa & $\mathbf{n}$ & $\%$ \\
\hline Instituição de ensino superior & 30 & 73,1 \\
Pública & 11 & 26,9 \\
Privada & & \\
Integrantes & 267 & 38,8 \\
Pesquisadores & 404 & 58,8 \\
Estudantes & 7 & 1,0 \\
Técnicos & 9 & 1,3 \\
Colaboradores estrangeiros & 687 & 100 \\
Total & 226 & - \\
Egressos & & \\
Área do conhecimento & 37 & 90,2 \\
Ciências da saúde; educação física & 2 & 4,8 \\
Ciências biológicas; fisiologia & 1 & 2,4 \\
Ciências da saúde; fisioterapia e terapia & & \\
ocupacional & & 2,4 \\
Ciências humanas; educação & 1 & \\
Linhas de pesquisa & & \\
1 a 3 & 26 & 63,4 \\
4 a 6 & 10 & 24,3 \\
Mais de 7 & 5 & 12,1 \\
\hline
\end{tabular}

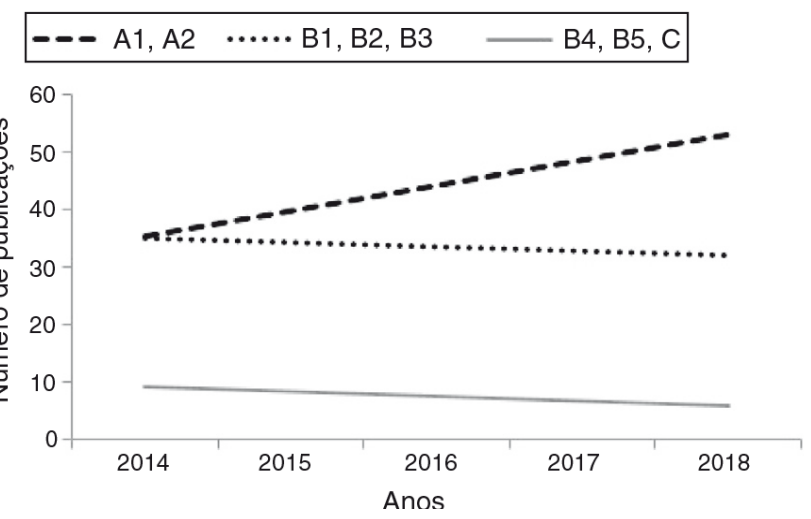

Figura 3. Tendência de qualidade das publicações dos GP em TF por ano.

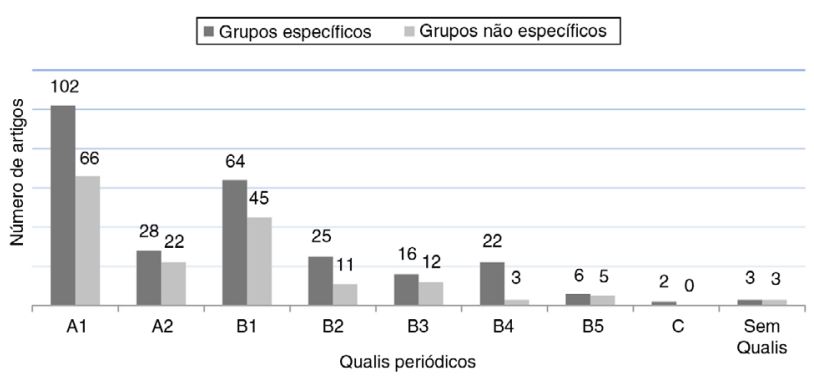

Figura 4. Classificação da produção dos GP específicos e não específicos em TF. 
No último quinquênio, 2017 foi o ano com o maior número de artigos publicados. Desde 2015 notase o predomínio de artigos no estrato A1, 2018 foi o ano com o maior número de artigos nesse estrato (Tabela 3).

A Figura 3 apresenta as tendências de qualidade das publicações dos líderes dos GP nos últimos cinco anos (2014-2018). Verifica-se tendência expressiva de aumento nas publicações em periódicos de maior qualidade (A1 e A2) e de queda na produção acadêmica em periódicos de qualidade mediana (B1, B2 e B3) e de qualidade inferior (B4, B5 e C) (Figura 3).

\section{Características das publicações}

Em relação à população nos estudos publicados sobre TF, observou-se que a maioria investigou praticantes de musculação, seguidos por idosos e adultos. As populações investigadas estão apresentadas na Figura 5. A nomenclatura usada para definir as populações ocorreu de acordo como descrito em cada artigo. Dentre as publicações, 41 foram estudos de revisão.
A partir da análise das palavras-chave identificaramse as $\mathbf{2 0}$ mais frequentes nos estudos publicados pelos líderes dos GP em TF no Brasil. O termo "treinamento de resistência" foi o mais citado, presente em 125 estudos, seguido por "treinamento de força" em 74, "força muscular" em 51, "exercício" em 44 e "exercício resistido" em 37. A Figura 6 ilustra as 20 palavras-chave mais citadas por meio de uma nuvem de palavras. O tamanho da fonte indica que há maior ou menor frequência de citações em relação às demais.

\section{DISCUSSÃO}

Este estudo teve como objetivo verificar e caracterizar a distribuição geográfica, evolução e produção científica dos GP em TF no Brasil. O presente trabalho tem caráter inovador, pois além das características dos GP traz análise da produção científica dos líderes, resultado importante, vista a escassez de análises da produção intelectual voltada para o TF. A década de 2010 destaca-se na formação de grupos de pesquisa no Brasil, com crescimento de $36 \%$ em seis anos. 0 mesmo pode-se observar nos

Tabela 3. Classificação da produção dos GP em TF, de acordo com o ano.

\begin{tabular}{ccccccccccc}
\hline \multirow{2}{*}{ Ano } & \multicolumn{10}{c}{ Estratos Qualis } \\
\cline { 2 - 13 } & A1 & A2 & B1 & B2 & B3 & B4 & B5 & C & s/ Q & Total \\
\hline 2014 & 16 & 14 & 21 & 9 & 8 & 5 & 4 & 1 & 0 & 78 \\
2015 & 35 & 11 & 8 & 6 & 5 & 5 & 2 & 0 & 0 & 72 \\
2016 & 32 & 13 & 27 & 6 & 8 & 5 & 0 & 0 & 1 & 92 \\
2017 & 39 & 9 & 41 & 9 & 1 & 8 & 4 & 1 & 4 & 116 \\
2018 & 44 & 7 & 11 & 3 & 4 & 3 & 0 & 0 & 1 & 73 \\
\hline
\end{tabular}

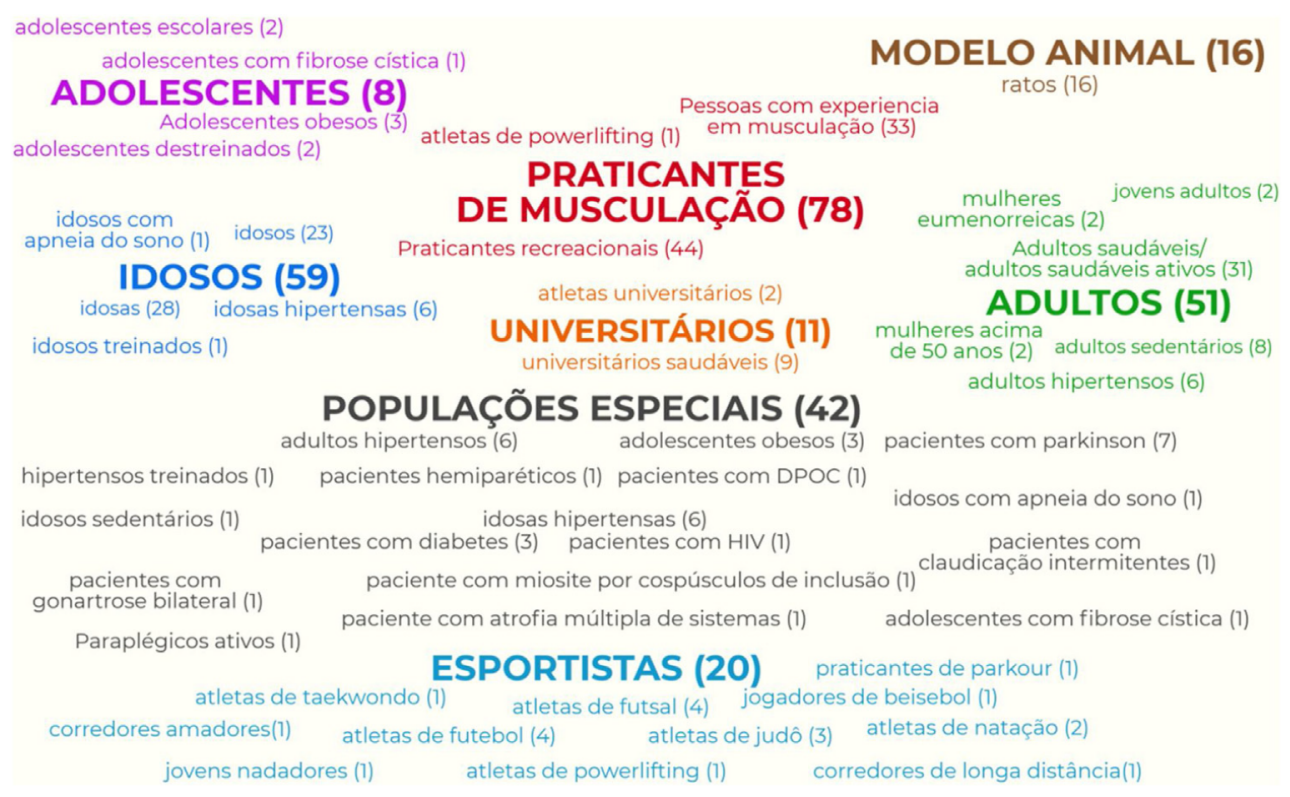

Figura 5. População investigada na produção científica dos líderes dos GP em TF (número de estudos). 


\section{pressão arterial \\ aptidão física força muscular \\ desempenho ocusãovasular hipertrofia muscular \\ treinamento de resistência \\ treinamento de força

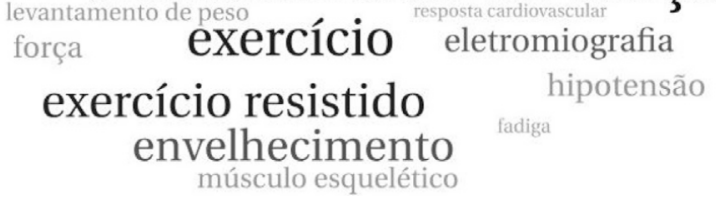

Figura 6. Principais palavras-chave dos estudos publicados pelos líderes dos GP em TF.

GP em TF, ocorre formação de GP em todos os anos dessa década. Tal crescimento é reflexo da expansão dos programas de pós-graduação stricto sensu na educação física, pois se trata da principal área à qual os GP estão vinculados.

O número de GP que investigam o TF no Brasil é mais elevado do que áreas como a psicologia do esporte e do exercício (29 GP) (Vilarino et al., 2017) e aproximase do número de GP de outras áreas, como atividade física e envelhecimento (40 GP) (Borges, 2012) e enfermagem (47 GP) (Backes et al., 2012). Entretanto, os dois últimos trabalhos foram publicados há mais de cinco anos e recomenda-se cautela na comparação.

A dependência do desenvolvimento de pesquisas a partir das instituições públicas ficou evidente nos GP em TF, assim como observado em outras áreas do conhecimento (Vilarino et al., 2017; Santos et al., 2011). Do mesmo modo, resultados semelhantes têm sido observados na literatura quanto à distribuição geográfica. Há concentração predominante na Região Sudeste, pois a área 21, na qual se encontra a educação física, tem elevado número de cursos de mestrado e doutorado na Região Sudeste (Brasil, 2013) em detrimento das regiões Nordeste, com apenas cinco programas de pós-graduação stricto sensu, e Norte, que não tem cursos de mestrado e doutorado. Com relação aos recursos humanos dos grupos, observa-se que os integrantes são predominantemente estudantes em relação ao número de técnicos administrativos e colaboradores estrangeiros (Vilarino et al., 2017).

A temática do TF tem a maioria de seus grupos de pesquisa vinculados à área das ciências da saúde, especificamente na educação física. Outra subárea das ciências da saúde que usa o TF em seu campo de atuação, especialmente na reabilitação e recuperação, é a fisioterapia e terapia ocupacional, entretanto essa apresentou apenas um GP. Apesar das evidências sobre os benefícios da prática de TF na manutenção da saúde e tratamento de doenças, esse é um tema pouco investigado nas demais áreas das ciências da saúde. Ressalta-se que essa temática não deve ficar restrita à educação física, necessitando de expansão e investigação das demais áreas de conhecimento que englobam as ciências da saúde.

A internacionalização da produção científica é o fator responsável pela tendência observada de publicação nos estratos de maior qualidade e redução nos estratos intermediário e inferior, ao longo dos cinco anos analisados. A classificação dos periódicos nos estratos mais elevados está relacionada a: 1) sua identidade na área, que foi estabelecida a partir da determinação de quatro indicadores de adesão (ADE1 a ADE4) por meio dos coordenadores dos programas; 2) ao fator de impacto, métrica obtida por meio da mediana do Journal Citation Reports (JCR) e do índice $\mathrm{h}$ do Scimago Journal \& Country Rank (SJR) que muitas vezes é maior em periódicos internacionais quando comparados com os nacionais; e 3) a indexação do periódico em base de dados (Capes, 2017). Na área 21, na qual a educação física está inserida, os periódicos classificados como A1 normalmente têm alta adesão na área e alto fator de impacto.

Entretanto, o novo Qualis (Coordenação, 2013b) proposto para o Quadriênio 2017-2020, pode alterar o modo de avaliação e por consequência a produção de conhecimento da área, fazer com que os pesquisadores, estudantes desde a graduação até o pós-doutorado, procurem publicar seus artigos em periódicos com alto fator de impacto e preferencialmente indexados nas bases de dados Web of Science e Scopus. Dessa forma, deverá ocorrer uma redução no número de artigos em português e aumentar significativamente os publicados em inglês, além de reduzir a quantidade de artigos publicados e em especial aqueles em periódicos sem indexação. Isso deverá dar maior qualidade, visibilidade aos conteúdos e pesquisas brasileiras no mundo, especialmente tratando-se de treinamento de força. Por outro lado, há risco de distanciamento entre as melhores publicações e os professores em sua prática, pois dificulta o acesso de 
docentes de escolas, academias e clubes, caracteriza aumento do abismo entre a universidade e essas instituições de intervenção. Ao mesmo tempo em que a mudança pode ser excelente do ponto de vista científico e acadêmico, surgem novos desafios em termos de impacto do conhecimento produzido na prática profissional.

Outra questão que deve ser destacada é que para manutenção e crescimento das publicações de alta qualidade na área são necessários recursos financeiros para o desenvolvimento das pesquisas, a fim de subsidiar as traduções e revisões para a língua inglesa, além do pagamento de taxas de publicação, característica presente em alguns periódicos. Para os GP receberem recursos financeiros de origem pública, a publicação em periódicos internacionais com fator de impacto tem sido exigência das principais agências financiadoras. Observa-se que o líder do Grupo de Estudo e Pesquisa em Adaptações Neuromusculares ao Treinamento de Forçada Universidade de São Paulo (USP) apresentou elevado número de artigos no estrato A1. Nesse sentido ressalta-se a importância das agências financiadoras. Nesse caso, é reconhecido que a Fundação de Amparo à Pesquisa do Estado de São Paulo (Fapesp) tem proporcionado um apoio financeiro superior ao feito pelas demais fundações estaduais. Além disso, a consolidação do grupo devese ao considerável número de integrantes e esse foi o segundo grupo criado no Brasil sobre o tema, em 2001.

Os critérios estabelecidos para avaliação dos líderes e dos GP na área da saúde têm ultimamente focado, sobretudo, na qualidade da produção intelectual, demonstrada pelo valor dado à publicação de artigos em periódicos nacionais ou internacionais que têm fator de impacto. Nesse sentido, o conteúdo do estudo não é fator determinante de qualidade do trabalho, e sim onde o periódico está indexado e suas métricas (Lazzarotti Filho et al., 2018). A queda na produtividade em estratos inferiores (B4 e B5) pode ser explicada pelo sistema de "travas", que limita a obtenção de elevado número de pontos nesses, força os pesquisadores a buscar periódicos com fator de impacto.

O uso de termos MeSH (Medical Subject Headings) mostrou-se predominante na maioria dos estudos, visto que as principais palavras-chave citadas são termos MeSH. Essa é uma exigência de grande parte dos periódicos, pois serve como terminologia-padrão para indexar literatura científica (Brandau et al., 2005).

A produção dos líderes mostra-se voltada para as populações de adultos e idosos. A relação entre TF e o envelhecimento mostrou-se como uma das dez áreas mais estudadas dentro do tema
(Kraemer et al., 2017). Em contrapartida, pouco foi produzido sobre adolescentes e crianças, essa última investigada em apenas um estudo. No entanto, observase uma maior procura de crianças e adolescentes para a prática de TF em academias. Essa lacuna em relação às populações estudadas deve ser tema de pesquisas futuras, a fim de oferecer respaldo científico para os profissionais da área. Destaca-se a variedade de publicações em populações especiais, como pessoas com hipertensão, obesidade e doença de Parkinson. Evidencia-se que o TF nessas e em outras populações especiais tem se mostrado efetivo em reduzir sintomas de doenças (Silva-Batista et al., 2017), melhorar componentes da aptidão física (Mynarski et al., 2014) e condições de saúde (Gambassi et al., 2017).

Devemos considerar que o uso exclusivo da base de dados do DGPB, mesmo que seja a principal base com informações sobre os GP brasileiros, pode não contemplar todos os grupos existentes, bem como a produção intelectual de outros líderes ou pesquisadores dentro das ciências do esporte, essa é uma limitação do presente estudo. Outra questão que deve ser destacada é que a forma de veiculação mais rápida do conhecimento produzido tem sido através da publicação de artigos em periódicos científicos, entretanto as publicações de livros e capítulos de livros sobre TF também têm importância, porém não foram analisadas.

Assim, os resultados deste estudo auxiliam os GP em TF em suas futuras pesquisas, pois evidenciam lacunas de conhecimento, podem servir de parâmetro para nortear novas pesquisas. Além disso, é fundamental para o crescimento da área uma autoanálise, na qual estudantes e profissionais possam ter conhecimento do que foi e do que é feito, permitirá assim um maior diálogo entre os GP e crescimento da área.

\section{CONCLUSÃO}

A maioria dos GPs que estuda o treinamento de força no Brasil é proveniente de instituições públicas e concentra-se na Região Sudeste. Destaca-se o Estado de São Paulo como referência da pesquisa em TF atualmente.

Os GP brasileiros que investigam o treinamento de força produzem conhecimento relevante e a qualidade da produção intelectual dos líderes está em evolução. A produção de conhecimento mostrou predomínio sobre a população de praticantes de musculação, adultos e idosos. Nos últimos cinco anos, observouse tendência expressiva de aumento nas publicações sobre o tema em periódicos de maior qualidade e queda de publicações em periódicos de qualidade mediana e inferior. 
O levantamento dos grupos de pesquisa no Brasil com análise da produção científica sobre determinado tema possibilita o conhecimento do estado da arte no campo de pesquisa. Além disso, a identificação da localização dos grupos permite o estabelecimento de novas parcerias institucionais para melhor qualidade na produção.

\section{FINANCIAMENTO}

Coordenação de Aperfeiçoamento de Pessoal de Nível Superior (Capes), Código de Financiamento 001.

\section{CONFLITOS DE INTERESSE}

Os autores declaram não haver conflitos de interesse.

\section{REFERÊNCIAS}

Backes VMS, do Prado ML, Lino MM, Ferraz F, Reibnitz KS, Canever BP.Grupos de pesquisa de educaçãoem enfermagem do Brasil. Rev Esc Enferm USP 2012;46(2):436-42.

Borges LJ, dos Santos SFdS, Scherer FC, Benedetti TRB. Grupos de pesquisa sobre atividade física e envelhecimento no Brasil. Rev bras ativ fís saúde 2012;17(2):114-20.

Brandau R, Monteiro R, Braile DM. Importância do uso correto dos descritores nos artigos científicos. Rev bras cir cardiovasc 2005;20(1):VII-IX.

Capes. Critérios de Classificação Qualis Área 21 -Educação Física, Fisioterapia, Fonoaudiologia e Terapia Ocupacional, 2017. Dispo- nível em: http://capes.gov.br/component/content/ article/44- avaliacao/4666-educacao-fisica.

Casonatto J, Goessler KF, Cornelissen VA, Cardoso JR, Polito $M D$. The blood pressure-lowering effect of a single bout of resistance exercise: a systematic review and metaanalysis of randomised controlled trials. Eur J Prev Cardiol 2016;23(16): 1700-14.

Conselho, Conselho Nacional de Desenvolvimento Científico e Tec- nológico. Diretório do Grupos de Pesquisa no Brasil. Disponível em: http://lattes.cnpq.br/web/dgp.

Conselho, Conselho Nacional de Desenvolvimento Científico e Tec- nológico. Plataforma Lattes: Diretório dos Grupos de Pesquisa no Brasil. Disponível em: http://lattes.cnpq.br/.

Coordenação de Aperfeiçoamento de Pessoal de Nível Supe-rior. Documento de área 2013. Brasília, DF: Capes, 2013. Disponível em: https://www.capes.gov.br/images/stories/ download/ avaliacaotrienal/Docs de area/Educa\%C3\%A7\%C3\% A3o doc area e comiss\%C3\%A3o 21out.pdf.

Coordenação de Aperfeiçoamento de Pessoal de Nível Superior. Documento de área 2018. Brasília, DF: Capes, 2013. Disponível em: https://sucupira.capes.gov.br/sucupira/public/ consultas/coleta/programa/quantitativos/quantitativoles. jsf ? areaAvaliacao $=21$ \&areaConhecimento $=40900002$.

Drenowatz C, Sui X, Fritz S, Lavie CJ, Beattie PF, Church TS, et al. The association between resistance exercise and cardiovascular disease risk in women. J Sci Med Sport 2015;18(6):632-6.
Esco MR. Resistance Training for Health and Fitness. In: Medicine ACoS, ed. American College of Sports Medicine. Indianapolis: American College of Sport Medicine; 2013:1-2.

Ferraz RRN, Barnabé AS, Quoniam L, Santos AMd, Mariosa DF. Aspec- tos históricos da criação dos grupos de pesquisa em dengue no Brasil com a utilização da ferramenta computacional ScriptGP. Ciênc Saúde Colet 2018;23:837-48.

Gambassi BB, Uchida MC, Sousa TMS, Schwingel PA, Pulcherio J, Almeida F. Effects of a new combined training approach on components of the functional autonomy of healthy elderly women. J Exerc Physiol Online 2017;20(3):45-52.

Ishiguro H, Kodama S, Horikawa C, Fujihara K, Hirose AS, Hirasawa $\mathrm{R}$, et al. In search of the ideal resistance training program to improve glycemic control and its indication for patients with type 2 diabetes mellitus: a systematic review and meta-analysis. Sports Med 2016;46(1):67-77.

Kraemer WJ, Ratamess NA, Flanagan SD, Shurley JP, Todd JS, Todd TC. Understanding the science of resistance training: An evolutionary perspective. Sports Med 2017;47(12):2415-35.

Kraschnewski JL, Sciamanna CN, Poger JM, Rovniak LS, Lehman $\mathrm{EB}$, Cooper $\mathrm{AB}$, et al. Is strength training associated with mortality benefits? A 15 year cohort study of US older adults. Prev Med 2016;87:121-7.

Lazzarotti Filho A, Mascarenhas F, Stigger MP, da Silveira R, Silva AM. Tendências no campo da educação física brasileira Análise dos documentos produzidos pela área 21 da Capes. Rev bras ciênc esporte 2018.

Marinho A, Barbosa-Rinaldi IP. Ginástica: reflexões sobre os gru- pos de pesquisa cadastrados no diretório do CNPq. J Phys Educ 2010;21(4):633-44.

Mynarski J, dos Santos L, Verffel A, Mello D, Berticell MW, Olkoski MM. Efeitos de diferentes programas de exercícios físicos sobre a composição corporal e a autonomia funcional de idosas com risco de fratura. J Phys Educ 2014;25(4):609-18.

Rhodes RE, Lubans DR, Karunamuni N, Kennedy S, Plotnikoff R. Factors associated with participation in resistance trai- ning: a systematic review. Br J Sports Med 2017, bjsports -2016-096950.

Santos SFdSd, Ferrari EP, Pacheco RL, Santos SGd, Benedetti TRB, Pires-Neto CS. Contributions of Kinanthropometry in Brazil: rese- arch groups and scientific production. Rev bras cineantropom desempenho hum 2011;13(4):306-12.

Silva-Batista C, de Brito LC, Corcos DM, Roschel H, de Mello MT, Piemonte ME, et al. Resistance training improves sleep quality in subjects with moderate Parkinson's disease. The J Strength Cond Res 2017;31(8):2270-7.

Teixeira FA, Marinho A. Atividades de aventura: reflexões sobre a produção científica brasileira. Motriz, Rio Claro 2010;16(3):536-48.

Thompson WR. Worldwide survey of fitness trends for 2018: the CREP edition. ACSMs Health Fit J 2017;21(6):10-9.

Vilarino GT, Dominski FH, Andrade RD, Felden ÉPG, Andrade A. Análise dos grupos de pesquisa em psicologia do esporte e do exercício no Brasil. Rev bras ciênc esporte 2017;39(4):371-9.

Westcott W. ACSM strength training guidelines: Role in body compo- sition and health enhancement. ACSM's Health \& Fitness Journal 2009;13(4):14-22. 